\title{
Online Monitoring Based Versatile Telemedical System
}

\author{
Miss. Neha A. Ghadage ${ }^{1}$, Mr. A.S. Tamboli ${ }^{2}$ \\ Student, Bachelor of Engineering, Electronics \& Telecommunication Engineering Department, Dr. Daulatrao Aher \\ College of Engineering, Karad, Maharashtra, India ${ }^{1}$ \\ Assistant Professor, Electronics \& Telecommunication Engineering Department, Dr. Daulatrao Aher College \\ Engineering, Karad, Maharashtra, India ${ }^{2}$
}

\begin{abstract}
Telemedicine is the use of telecommunication technology in medical field. This system consists of both hardware and software component at the patient and doctor ends. The proposed project in this paper provides an image based technique to analyse continuous monitoring of ECG signal and movement of patient by using sensors and digital camera. The system consist of sensors, software (MATLAB), microcontroller, data acquisition unit (computer).The proposed system uses android mobile phone as a interlink between patient and consulting doctor. This method captures the ECG signal and movement of patient from ICU monitoring machine using a digital camera and transmit the data through internet. The prime goal was to develop a telemedical system so that healthcare professional can monitor their patient continuously. The paper proposes a method to capture the image of patient condition using ECG and make the capture image be available to the physician. This system provide information about medical status of patient.
\end{abstract}

Keywords: Telemedicine, ECG, MATLAB, Android, Sensors, Patient Monitoring System.

\section{INTRODUCTION}

Health is very important for human being [2]. advances in communication facilitates. The specialist Telemedicine is highly interested and used in hospitals, doctor and the patient separated by thousands of clinics, nursing homes, rehabitation hospitals, homes, kilometers use mobile App to continuous monitoring of schools developed area for purpose of monitoring ECG signal and movement of patient [1]. Careful design increase patients comfort and improve living standard of of hardware and software component of the system is able patients[4].Tele-health system have many applications like to fulfill any further requirement of users [2]. prisons or health department[3].

The healthcare professionals play the major role in day to day life[2].The main aim of this paper is to develop continuous monitoring ECG signal and movement of patient [3].Every time it is not possible for doctors to monitor the patient hence we develop android phone based monitoring system [2].It include anything like medical services at the inpatient or outpatient stage. The patients who live in rural areas they not get proper medical service without any transportation barriers. So we can proposed telemedical system [3].

\section{BACKGROUND}

Rahmat Sanudin- proposed LabVIEW based monitoring system for small scale application. The system is built using laboratory virtual instrumentation engineering workbench (LabVIEW) software and setting appropriate Internet Protocol(IP) address as well as physical connection between two terminals. This system is able to exchange mobile text message. The drawback of the system is that it is useful for only small scale application [1].

Even a simple mobile phone can become a powerful Real time wireless health monitoring application using healthcare device. Today "Smartphone" plays important mobile device by using this system the information role in transmit medical information and advice. contained in the text or email message. The healthcare Smartphone's will be the most popular technological professional can provide necessary treatment for patient development for the doctors[2].

Here we are trying to implement portable monitoring system with android mobile phone in which acquire ECG signal are processed[3]. Telemedicine reduces the cost of healthcare as well as increase the efficiency of patient life .Telemedicine facility together with technological

[2].A portable wireless ECG monitoring system using GSM technique with real time detection of beat abnormalities.

This paper proposes the design of a real time low cost portable wireless ECG acquisition system which implemented through common mobile phone and high end 
recorder [3].Wearable ECG recording and monitoring The proposed project in this paper provide image based system based on MSP430 microcontroller. The system technique to analyse continuous monitoring of ECG signal uses instrumentation amplifier and ultralow power through digital camera for image capturing and analysis electrode to record the biosignal. body temperature and blood pressure. microcontroller for the storage SD card. They use ECG

The patient and physician need to be stationary for the entire process [4]. Aim of this project is to implement and develop ECG monitoring system based on smart phone. It is low cost heart rate monitoring solution. The application of this project is realization of ECG data signal which is sent from heart rate monitoring device [5].

The sensors used to measure various parameter such as

\section{III.BLOCK DIAGRAM}

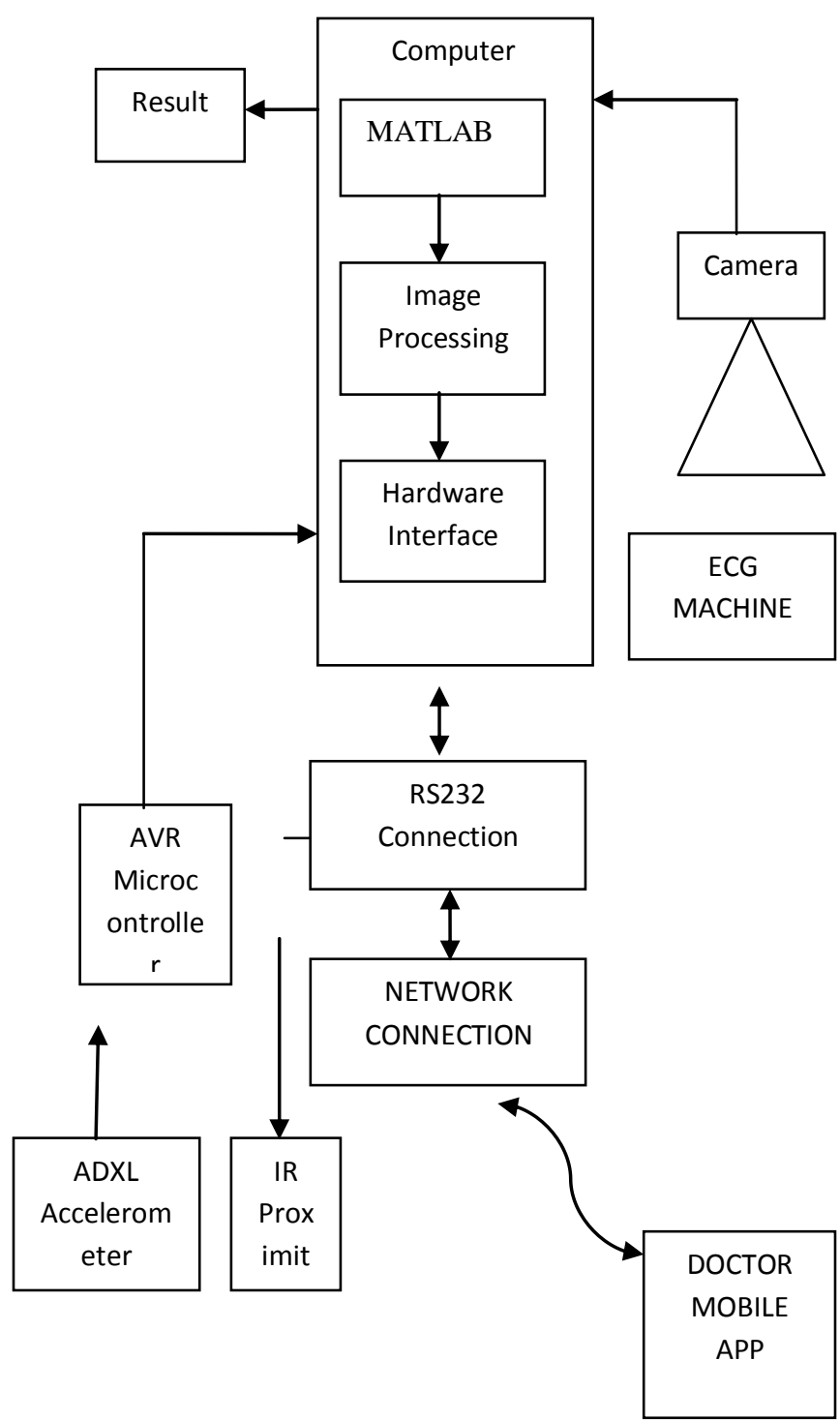
performed MATLAB tool as well as data sending system. This image is available to related doctor by using android cell phone. Cardiology is important area to showing major growth in telemedicine [6].

In the telemedical system ECG machine is the major part. ECG machine is used for graphically recording and monitoring the electrical activity of heartbeat. FPGA (Field Programmable Gate Array) based USB camera is used for capturing the image of ECG (Electrocardiogram) machine.

Captured image is given to computer through wired connection. Computer consist of MATLAB, Image processing and hardware interface. The captured image is streamed into MATLAB where it is processed and given to image processing. The system uses sensors like ADXL Accelerometer sensor and IR Proximity sensor.IR proximity sensor able to detect presence of nearby object. ADXL (Advanced Digital Subscriber Line) accelerometer sensor is used for detection of object position.

AVR (Advanced Virtual RISC) microcontrollers used for storing sensor data.AVR microcontroller have large memory. This data is given to computer. The whole result of computer is stored in result block. This whole data is given to network connection through RS232 connection. In Tele communication RS232 is standard for serial communication transmission of data.

By using server this data is available at doctor android mobile app. Android mobile app is used to display ECG image and movement of patient. The capturing of image by using real time operating digital FPGA based USB camera and saving this image in computer.

Then the system consists of a series of image processing technique that perform image conversion and image comparison. The processed image is converted in to text document and it is uploaded to server by using web application.

Hence any change in the ECG signal of any patient which is present in ICU then notification is send to the doctor phone. Image processing is a method to convert image into digital form and perform some operations on it, in order to get and enhanced image or to extract some useful information from it. AVR was one of the first microcontroller families to use on chip flash memory for program storage. AVR microcontroller finds many applications as embedded systems, they are also used in the popular Adriano line of open source board designs.

\section{FLOWCHART}

Fig.1. Block Diagram of Proposed System
Following figure shows the flow of model. 
IARJSET

Vol. 4, Special Issue 2, January 2017

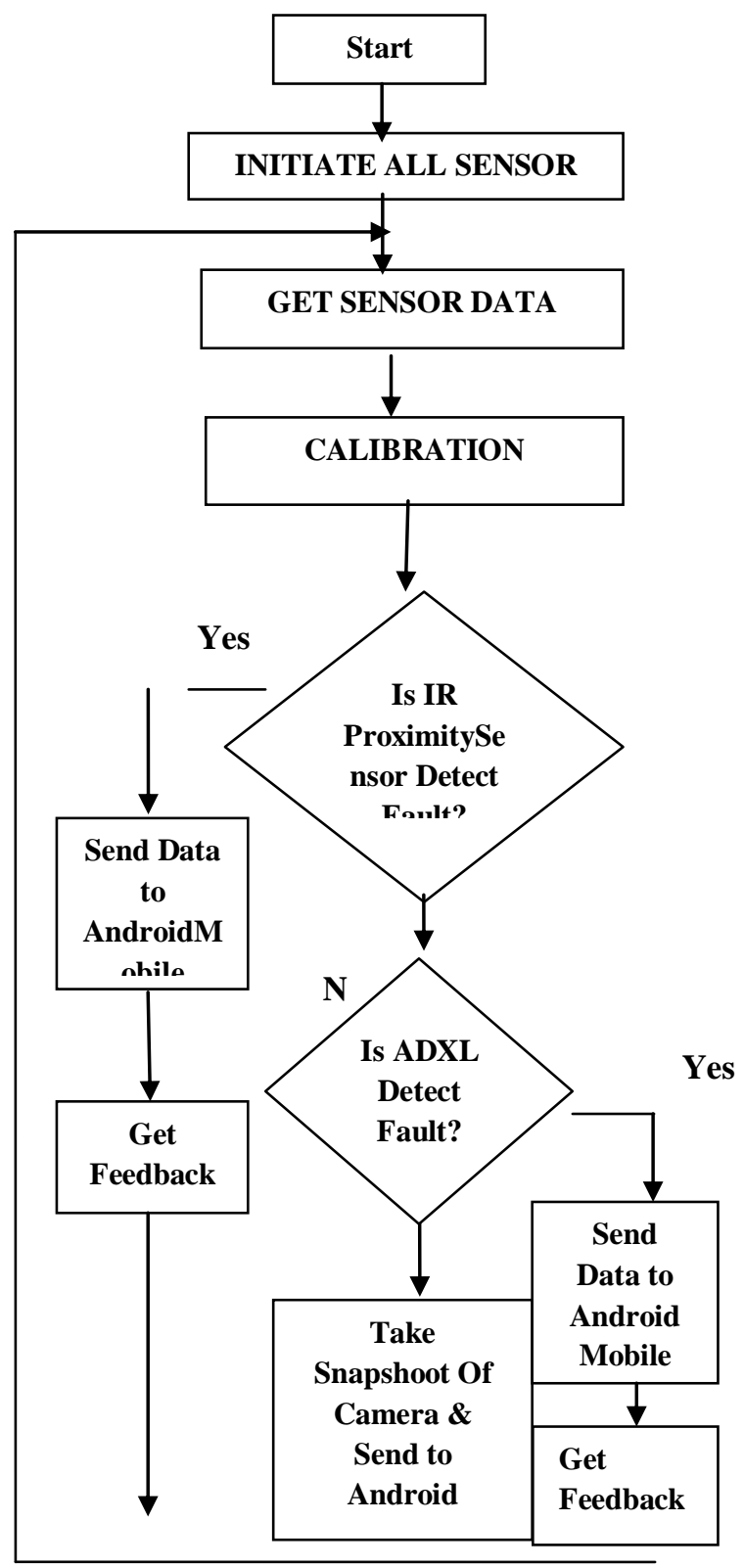

Fig.2. Flow chart

\section{V.CONCLUSION}

The project online monitoring based versatile telemedical system is a telemedicine application which is used by doctors for continuous monitoring of patient. Here, healthcare professional can monitor their patient even if he is not present near the patient. Our system is simple and power efficient. It is easy to use, safe, fast and accurate.

\section{REFERENCES}

[1] Rahmat Sanudin, Member,IEEE, Mohd Helmy Abd Wahat Member, IEEE, and Wan Suhaimizan Wan Zaki "Labview-base Monitoring System for Small-scale Application",MASAUM Journal of Basic and Applied Sciences, 2009
[2] Amna Abdullah, Asma Ismael, Aisha Rashid, Ali Avou-EINour, and Mohammed Tarique "Real Time Wireless Health Monitoring Applicaton Using Mobile Devices",Dept.of Electrical Engineering, Ajman University of Sciences and Technology, Fujairah, United Arab Emirates International Journal of Computer Networks and Communications (IJCNC), 2015

[3] Dr. (Mrs.) R. Sukanesh, S. Veluchamy, M. Karthikyn, “A Portable Wireless ECG Monitoring System using GSM Technique with Real Time Detection of Beat Abnormalities ",Dept. of Electronics and Communication Engineering, TCE,Madhurai and Dept. of Electronics and Communication Engineering, AUT, Madhurai, International Journal of Engineering Research, 2014

[4] Asifiqbal Thakor, ProfessorRahul Kher and ProfessorDipak Patil, "Wearable ECG Recording and Monitoring System based onMSP430 Microcontroller",International Journal of Computer Science and Telecommunication, 2012

[5] Ayaj Akram ,Raheel Javed, Awais Ahmad," Android Based ECG Monitoring System", Department of Electrical Engineering ,University of Engineering and Technology Lahore, Pakistan , International Journal of Science and Research(IJSR)

[6] M. V. M. Figueredo, J.S. Dias, "mobile telemedicime system for home care and patient monitoring ", Laboratoria de Information em Saude, Pontificia Universidade Catolica do Parana, Curitiba, Parana, Brazil, Program de Pos-Graduacao em Technologia em Saude, Pontifica Universidade Catolica do Parana, Caritiba, Parana,Brazil

[7] Yahia Said, Taoufik saidani, Fethi Smach, Mohamed Atri ,"An ECG -Based Smart Camera Sytem", Laboratory of Electronics And Microelectronics Faculty of Science of Monastir, TUNISIA

[8] Harsha G.S.'Design An Implementation Of Online Patient Monitoring System", Department of Electronics \& communication, Channabasaveshwara Institute of Technology, Gubbi, India 